Research Article

\title{
Penetration Effect: Exotic Behavior of a Wave in Anisotropic Media
}

\author{
K. Vytovtov and O. Pischin \\ Astrakhan State Technical University, Tatischeva 16, Astrakhan 414056, Russia \\ Correspondence should be addressed to K. Vytovtov; vytovtov_konstan@mail.ru
}

Received 15 February 2017; Revised 26 March 2017; Accepted 2 May 2017; Published 23 May 2017

Academic Editor: Mustapha C. E. Yagoub

Copyright (C) $2017 \mathrm{~K}$. Vytovtov and O. Pischin. This is an open access article distributed under the Creative Commons Attribution License, which permits unrestricted use, distribution, and reproduction in any medium, provided the original work is properly cited.

Plane harmonic wave propagation along an interface between vacuum and a semi-infinite uniaxial anisotropic medium is considered. It is shown that there is a bulk wave within an anisotropic medium in this case. It is also proved for the first time that a reflected wave must propagate perpendicularly to an interface. Moreover, a reflected wave is absent in the case of ordinary wave propagation.

\section{Introduction}

A phenomenon of plane electromagnetic wave propagation in vacuum parallel to an interface between vacuum and an anisotropic medium has been considered in [1]. It has been shown analytically in that paper for the first time that a bulk wave can be excited within an anisotropic medium in the considered case. Also this phenomenon has been defined as "penetration effect" for the first time. It has been obtained that a real part of the Poynting vector normal component of a wave propagating in an anisotropic medium is not equal to zero when an incident wave propagates tangentially to an interface between the media. This fact gives us the possibility of talking about electromagnetic wave propagation within an anisotropic medium under an oblique angle. In addition, the authors have suggested the possible practical applications of this effect. In particular, it has been proposed to use these structures to construct frequency detectors and power dividers for microwave communication systems. However, such an important point as behavior of a reflected wave has not been considered in [1]. The hypothesis about reflected wave propagation perpendicular to an interface has been presented in [2]. but this problem has not been studied in detail. For example, in that work, a deep derivation of the expressions for the reflection and transmission coefficients has not been presented. The physical effect in [2] has not been also substantiated.
It is obvious that studying behavior of the transmitted and reflected waves when an incident wave propagates parallel to an interface between anisotropic and isotropic media has important theoretical and practical significance. Indeed this issue has not been studied theoretically despite the fact that a similar phenomenon has been described even in hydrodynamics [3]. From a practical point of view, this question is important as this phenomenon leads to additional power losses. Indeed, earlier, power losses determined by "penetration effect" have not been taken into account. Furthermore, penetration effect allows us to design new optical and microwave devices.

In this paper, we consider the ideal case of wave propagation over an infinite interface between free space and a uniaxial dielectric anisotropic medium without frequency dispersion. An orientation of the anisotropy axis is taken arbitrary in our treatment. At the same time, we do not study the problem of the possibility of exciting such a wave and the grounds for this assumption. It is important to note that this particular case cannot be described by using wellknown methods [4] as the transmission coefficient would be zero if the classical theory was used. However, in accordance with the solution of Maxwell's equations, there is a transmitted wave in the anisotropic medium. Obviously, the results presented in this study require further experimental verification. However, at the same time, all expressions 
are obtained directly from Maxwell's equations under the classical boundary conditions for the field and wave vector components by using accurate analytical transformations only. These facts confirm the correctness of the presented results.

The behavior of both transmitting in the anisotropic medium and reflecting in vacuum waves is investigated in this paper too. It is shown analytically that a reflected wave should propagate perpendicularly to the interface to satisfying Maxwell's equations and the boundary conditions simultaneously. The expressions of reflection and transmission coefficients for a semi-infinite medium are obtained and the analysis of these coefficients is carried out.

\section{Statement of the Problem}

In this paper, we study the particular case of the uniaxial anisotropic dielectric medium with the scalar permeability $\mu$ and the permittivity dyadic in the following form:

$$
\overleftrightarrow{\varepsilon}=\left|\begin{array}{ccc}
\varepsilon_{11} & 0 & 0 \\
0 & \varepsilon_{11} & 0 \\
0 & 0 & \varepsilon_{33}
\end{array}\right|
$$

For example, it can be a plasma medium near the resonance region [5] when external bias field is small and therefore the off-diagonal components $\varepsilon_{12}$ and $\varepsilon_{21}$ in (1) can be negligible.

It is assumed that the anisotropy axis is orientated in the incidence plane under an arbitrary angle $\beta$ with the normal to the interface (Figure 1). Now using Euler transformation [6], we can write the electric permittivity dyadic in the $x y z$ system as follows:

$$
\begin{aligned}
\overleftrightarrow{\varepsilon} & =\left|\begin{array}{ccc}
\varepsilon_{x x} & 0 & 0 \\
0 & \varepsilon_{y y} & \varepsilon_{y z} \\
0 & \varepsilon_{z y} & \varepsilon_{z z}
\end{array}\right| \\
& =\left|\begin{array}{ccc}
\varepsilon_{11} & 0 & 0 \\
0 & \varepsilon_{33} \sin ^{2} \beta+\varepsilon_{11} \cos ^{2} \beta & \left(\varepsilon_{33}-\varepsilon_{11}\right) \cos \beta \sin \beta \\
0 & \left(\varepsilon_{33}-\varepsilon_{11}\right) \cos \beta \sin \beta & \varepsilon_{11} \sin ^{2} \beta+\varepsilon_{33} \cos ^{2} \beta
\end{array}\right| .
\end{aligned}
$$

The geometry of the problem is presented in Figure 1. Here $x^{\prime} y^{\prime} z^{\prime}$ is the coordinate system connected with the permittivity dyadic (1) ( $z^{\prime}$ is the anisotropy axis); $x y z$ is the coordinate system connected with the normal to the interface, where $z$ is a normal to the interface.

In the considered case, a plane harmonic wave with the wavenumber $k_{\text {inc }}=k_{y}=\omega \sqrt{\mu_{0} \varepsilon_{0}}$ propagates above the interface, where $\omega$ is the frequency, $\varepsilon_{0}$ is the dielectric permittivity of vacuum, and $\mu_{0}$ is the magnetic permeability of vacuum.

\section{Maxwell's Equations}

The solutions of Maxwell's equations [4] for the considered geometry can be taken in the exponential form

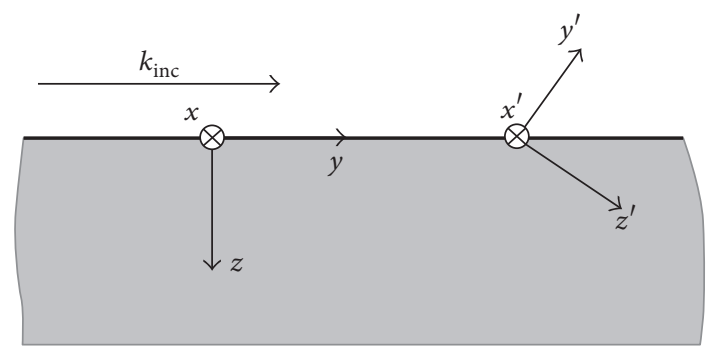

FIGURE 1: Geometry of the problem.

$\exp \left(j \omega t-j k_{x} x-j k_{y} y-j k_{z} z\right)$. Thus Maxwell's equations [2] in the scalar form are written as follows:

$$
\begin{aligned}
-j k_{y} H_{z}+j k_{z} H_{y} & =j \omega \varepsilon_{x x} E_{x}, \\
-j k_{z} H_{x} & =j \omega \varepsilon_{y y} E_{y}+j \omega \varepsilon_{y z} E_{z}, \\
j k_{y} H_{x} & =j \omega \varepsilon_{z y} E_{y}+j \omega \varepsilon_{z z} E_{z}, \\
-j k_{y} E_{z}+j k_{z} E_{y} & =-j \omega \mu_{0} H_{x}, \\
-j k_{z} E_{x} & =-j \omega \mu_{0} H_{y}, \\
j k_{y} E_{x} & =-j \omega \mu_{0} H_{z} .
\end{aligned}
$$

Here, it is taken into account that

$$
\begin{aligned}
\operatorname{rot} H= & \left(\frac{\partial H_{z}}{\partial y}-\frac{\partial H_{y}}{\partial z}\right) \vec{x}+\left(\frac{\partial H_{x}}{\partial z}-\frac{\partial H_{z}}{\partial x}\right) \vec{y} \\
& +\left(\frac{\partial H_{y}}{\partial x}-\frac{\partial H_{x}}{\partial y}\right) \vec{z} \\
= & \left(-j k_{y} H_{z}+j k_{z} H_{y}\right) \vec{x}+\left(-j k_{z} H_{x}+j k_{x} H_{z}\right) \vec{y} \\
& +\left(-j k_{x} H_{y}+j k_{y} H_{x}\right) \vec{z} .
\end{aligned}
$$

Since the statement of the problem is shown in Figure 1, we have $k_{x}=0$. Therefore,

$$
\begin{aligned}
\operatorname{rot} H= & \left(-j k_{y} H_{z}+j k_{z} H_{y}\right) \vec{x}+\left(-j k_{z} H_{x}\right) \vec{y} \\
& +\left(j k_{y} H_{x}\right) \vec{z}
\end{aligned}
$$

The analogous transformations can be presented for the electrical field.

The solutions of (3) for this case give us two independent waves: an ordinary wave containing $\mathrm{H}_{y^{-}}, \mathrm{H}_{z^{-}}, \mathrm{E}_{x^{-}}$ components and an extraordinary wave containing the $H_{x^{-}}$,

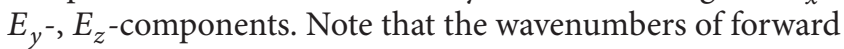
and backward waves are equal [2] for the ordinary wave, and these wavenumbers are not equal for the extraordinary wave. Now let us consider the behavior of these waves in detail. 


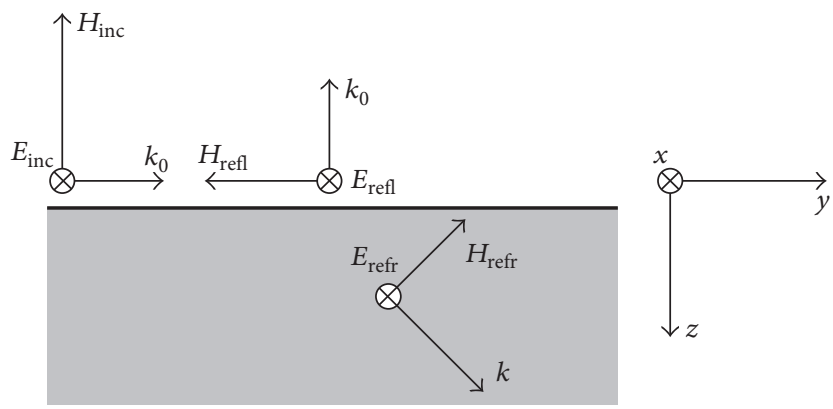

FIGURE 2: Geometry of the problem of the propagation and reflection of an ordinary wave.

\section{Ordinary Wave}

The geometry of the considered problem is shown in Figure 2. Using the first, fifth, and sixth equations of (3), we obtain the relations between the components of the electromagnetic fields for an ordinary wave (a wave containing $H_{y^{-}}, H_{z^{-}}, E_{x^{-}}$ components) in an anisotropic medium:

$$
\begin{aligned}
& H_{y}=\frac{1}{\rho_{0}} \sqrt{\frac{\varepsilon_{x x}}{\varepsilon_{0}}-1} E_{x}, \\
& H_{z}=-\frac{1}{\rho_{0}} E_{x},
\end{aligned}
$$

where $\rho_{0}=\sqrt{\mu_{0} / \varepsilon_{0}}$ is wave impedance of vacuum.

It is important that the normal magnetic field component $H_{z}$ and the tangential electric field component $E_{x}$ do not depend on the frequency and an inclination angle of the anisotropy axis. The frequency dependence of the tangential component of the magnetic field $H_{y}$ arises only in the case of frequency dispersion of the electric permittivity components in (1) [5].

Let us find the normal component of the wave vector $k_{z}$. Taking into account the continuity conditions for the tangential components of the wave vector, we obtain $k_{y}=k_{0}$. The normal component of the wave vector in an anisotropic medium is found by substituting (4) into the first equation in (3):

$$
\begin{aligned}
k_{z} & =\omega \sqrt{\frac{\varepsilon_{0}}{\varepsilon_{x x}-\varepsilon_{0}}}\left(\varepsilon_{x x} \sqrt{\frac{\mu_{0}}{\varepsilon_{0}}}-\sqrt{\varepsilon_{0} \mu_{0}}\right) \\
& =\omega \sqrt{\mu_{0}} \sqrt{\varepsilon_{x x}-\varepsilon_{0}} .
\end{aligned}
$$

It is followed from (7) that the refraction angle is

$$
\alpha=\arctan \frac{k_{y}}{k_{z}}=\arctan \sqrt{\frac{\varepsilon_{0}}{\varepsilon_{x x}-\varepsilon_{0}}} .
$$

The Poynting vector components are calculated by using the well-known method [4]:

$$
S_{z}=E_{x} H_{y}^{*}=\frac{1}{\rho_{0}} \sqrt{\frac{\varepsilon_{x x}}{\varepsilon_{0}}-1} H_{x}^{2}
$$

$$
S_{y}=-E_{x} H_{z}^{*}=\sqrt{\frac{\varepsilon_{0}}{\mu_{0}}} H_{x}^{2}=-\frac{1}{\rho_{0}} H_{x}^{2} .
$$

Thus the ordinary wave has both the real normal component of the wave vector (7) and the real normal component of the Poynting vector (9). Consequently the bulk wave must exist in an anisotropic medium. And now the power transmission angle (see (9) and (10)) is

$$
\alpha_{S}=\arctan \frac{S_{y}}{S_{z}}=\arctan \sqrt{\frac{\varepsilon_{0}}{\varepsilon_{x x}-\varepsilon_{0}}} .
$$

It is seen from (8) and (11) that $\alpha_{S}=\alpha$ for the considered wave type. Therefore, both the power flow and the wave front propagate in the same direction analogously to a usual isotropic medium.

\section{Extraordinary Wave}

The geometry of the considered problem is presented in Figure 3. Solving the second and third equations of (3) simultaneously, we obtain the relations between the field components for the extraordinary wave $\left(E_{x}, H_{y}, H_{z}\right)$ :

$$
\begin{aligned}
& E_{y}=-\frac{k_{z} \varepsilon_{z z}+k_{y} \varepsilon_{y z}}{\omega\left(\varepsilon_{y y} \varepsilon_{z z}-\varepsilon_{y z} \varepsilon_{z y}\right)} E_{x}=\chi_{y} H_{x}, \\
& E_{z}=\frac{k_{y} \varepsilon_{y y}+k_{z} \varepsilon_{z y}}{\omega\left(\varepsilon_{y y} \varepsilon_{z z}-\varepsilon_{y z} \varepsilon_{z y}\right)} E_{x}=\chi_{z} H_{x} .
\end{aligned}
$$

The normal component of the wave vector can be found by substituting the expressions (12) and (13) in the fourth equation of (3) that is equal to

$$
\begin{aligned}
k_{z} & =-\frac{k_{y}\left(\varepsilon_{y z}+\varepsilon_{z y}\right)}{2 \varepsilon_{z z}} \\
& \pm \sqrt{\frac{k_{y}^{2}\left(\varepsilon_{y z}+\varepsilon_{z y}\right)^{2}}{4 \varepsilon_{z z}^{2}}-\frac{k_{y}^{2} \varepsilon_{y y}-\omega^{2} \mu_{0}\left(\varepsilon_{y y} \varepsilon_{z z}-\varepsilon_{y z} \varepsilon_{z y}\right)}{\varepsilon_{z z}}} \\
& =\frac{\omega \sqrt{\mu_{0}}\left[-\sqrt{\varepsilon_{0}}\left(\varepsilon_{y z}+\varepsilon_{z y}\right)\right.}{2 \varepsilon_{z z}} \\
& \left. \pm \sqrt{\varepsilon_{0}\left(\varepsilon_{y z}+\varepsilon_{z y}\right)^{2}-4 \varepsilon_{z z}\left[\varepsilon_{0} \varepsilon_{y y}-\left(\varepsilon_{y y} \varepsilon_{z z}-\varepsilon_{y z} \varepsilon_{z y}\right)\right]}\right] .
\end{aligned}
$$




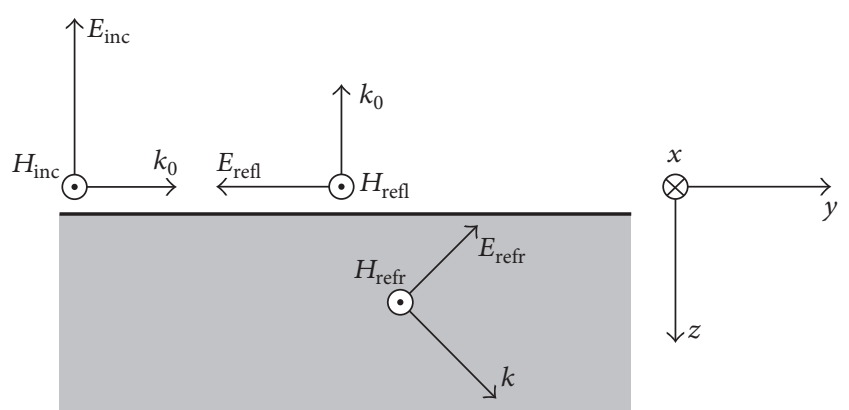

Figure 3: Geometry of the problem of the propagation and reflection of an extraordinary wave.

The forward wave is described by (13) with a "plus" and the backward wave with a "minus." Obviously, the angle between wave front propagation and the normal is

$$
\alpha=\arctan \frac{k_{y}}{k_{z}} .
$$

The Poynting vector components of the extraordinary wave are equal to

$$
\begin{aligned}
& S_{z}=E_{x} H_{y}^{*}=-\frac{k_{z} \varepsilon_{z z}+k_{y} \varepsilon_{y z}}{\omega\left(\varepsilon_{y y} \varepsilon_{z z}-\varepsilon_{y z} \varepsilon_{z y}\right)} E_{x}^{2}, \\
& S_{y}=-E_{x} H_{z}^{*}=-\frac{k_{y} \varepsilon_{y y}+k_{z} \varepsilon_{z y}}{\omega\left(\varepsilon_{y y} \varepsilon_{z z}-\varepsilon_{y z} \varepsilon_{z y}\right)} E_{x}^{2},
\end{aligned}
$$

where $H_{y}^{*}$ is the complex conjugate of $H_{y}$. The angle between the electromagnetic power flow propagation and a normal to the interface is as follows:

$$
\alpha_{S}=\arctan \frac{S_{y}}{S_{z}}=\arctan \frac{k_{y} \varepsilon_{y y}+k_{z} \varepsilon_{z y}}{k_{z} \varepsilon_{z z}+k_{y} \varepsilon_{y z}} .
$$

The analysis of the obtained expressions shows that the transmitted wave has the real normal (13) and tangential (16) components of the Poynting vector. Therefore the power of this wave propagates under an oblique angle in an anisotropic medium. The power propagation angle (15) does not coincide with the wave front propagation angle (14).

\section{Behavior of a Reflected Wave}

It has been shown earlier that a plane harmonic wave propagating parallel to an interface between vacuum and an anisotropic medium can excite a bulk wave propagating under an arbitrary angle within an anisotropic medium. Now it is necessary to describe behavior of a reflected wave in vacuum if it existed.

Let us take into account the fact that the tangential components of the wavenumbers of incident, reflected, and refracted waves must be equal one to other $k_{\text {inc }}=$ $k_{y \text { refr }}=k_{y \text { refl }}$ in accordance with Snell's Law [4] and these wavenumbers are also equal to the wavenumber in vacuum $k_{0}=\omega \sqrt{\varepsilon_{0} \mu_{0}}$. Then a reflected wave must propagate along an interface or perpendicular to an interface. Otherwise the frequency of a reflected wave must be more than the frequency of an incident wave to satisfy the boundary conditions. Indeed if a reflected wave is propagating under an oblique angle and the tangential component is equal to the wavenumber in vacuum then $k_{\text {refl }}=\sqrt{k_{y}^{2}+k_{z}^{2}}=\sqrt{k_{0}^{2}+k_{z}^{2}}>$ $k_{0}$.

Moreover a refracted wave has the tangential component of magnetic field for an ordinary wave and the tangential component of the electric field for an extraordinary wave. Therefore such component must be in a reflected wave as this component is absent in an incident wave. But such phenomenon is impossible when a reflected wave propagates along an interface. Based on these considerations, we assume that a reflected linearly polarized wave should propagate perpendicular to an interface between vacuum and an anisotropic medium.

\section{Coefficients of Propagation and Reflection of an Ordinary Wave}

Let us consider the ideal case of plane harmonic wave propagation above an interface between vacuum and a uniaxial anisotropic medium. It follows from (6) that a transmitting wave has the components $H_{y}, H_{z}, E_{x}$. Thus a reflected wave must also contain the $H_{y}$-component as this is absent in an incident wave.

The geometry of the problem is shown in Figure 2. Here $k_{0}$ is the wavenumber in vacuum; $H_{\text {inc }}, E_{\text {inc }}$ are the field components of an incident wave propagating along an interface; $H_{\text {refl }}, E_{\text {refl }}$ are the field components of the reflected wave; $H_{\text {refr }}, E_{\text {refr }}$ are the field components of the transmitting wave.

Let us write the continuity conditions for the field components at the interface between vacuum and an anisotropic medium [4]:

$$
\begin{aligned}
E_{\text {inc }}+E_{\text {refl }} & =E_{x \text { refr }}, \\
-H_{y \text { refl }} & =H_{y \text { refr }}+J, \\
B_{z \text { inc }} & =B_{z \text { refr }} .
\end{aligned}
$$

Take into account the fact that, in free spaces $H_{y}=\left(1 / \rho_{0}\right) E_{x}$ and $H_{z}=-\left(1 / \rho_{0}\right) H_{x}$, we can obtain from (18)

$$
\begin{aligned}
1+R & =T, \\
-\frac{1}{\rho_{0}} R & =\chi_{y} T+\frac{J}{E_{\text {inc }}}, \\
-\frac{\varepsilon_{0}}{\rho_{0}} & =\varepsilon_{0} \chi_{z} T .
\end{aligned}
$$

Here $\rho_{0}=\sqrt{\mu_{0} / \varepsilon_{0}}$ is the impedance of vacuum, $J$ is the surface current density at the interface, $R=E_{x \text { refl }} / E_{x \text { inc }}$ is the reflection coefficient, and $T=E_{x \text { refr }} / E_{x \text { inc }}$ is the transmission coefficient. Now we can find the transmission 


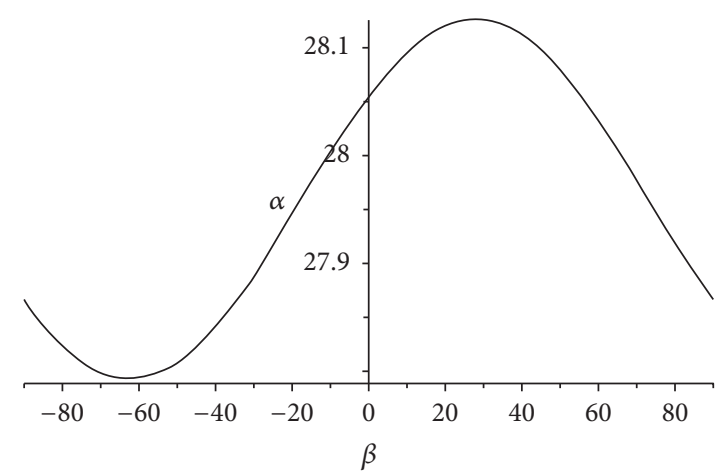

(a)

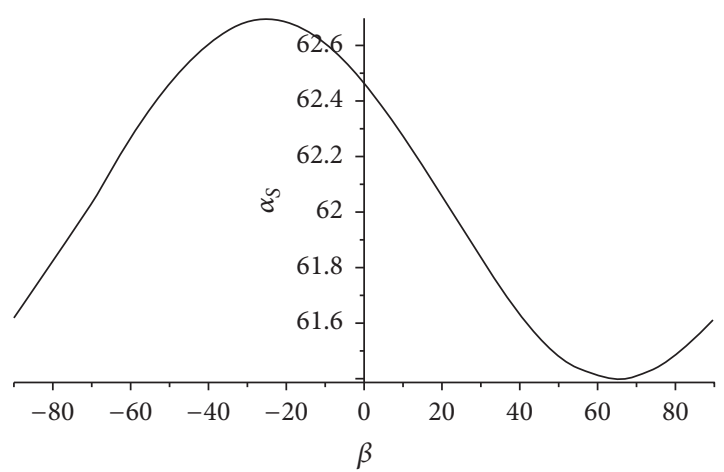

(b)

Figure 4: The dependencies of the transmission angles on the inclination angle.

and reflection coefficients, as well as the surface charge density:

$$
\begin{aligned}
& T=1, \\
& R=0, \\
& J=-\rho_{0}^{2} E_{\text {inc }} .
\end{aligned}
$$

Thus, if a harmonic plane wave containing the components $H_{y}, H_{z}, E_{x}$ propagates along an interface between vacuum and an anisotropic medium, then there is no reflected wave (21), the transmission coefficient is equal to one (20), and also there are the surface currents (22) at the interface. These currents are actually bias currents and these are associated with the molecule polarization phenomenon in an electromagnetic field [4].

\section{Coefficients of Transmission and Reflection of an Extraordinary Wave}

The geometry of the problem is given in Figure 3. The boundary conditions for the electromagnetic field components in this case are as follows:

$$
\begin{aligned}
& H_{x \text { inc }}+H_{x \text { refl }}=H_{x \text { refr }}, \\
&- E_{y \text { refl }}=E_{y \text { refr }}, \\
&-\varepsilon_{0} E_{z \text { inc }}=\varepsilon_{z y} E_{y \text { refr }}+\varepsilon_{z z} E_{z \text { refr }}+\sigma .
\end{aligned}
$$

Taking into account the relations $E_{x}=-\rho_{0} H_{y}, E_{x}=\rho_{0} H_{z}$ [4] and introducing the reflection $R=H_{x \text { refl }} / H_{x \text { inc }}$ and transmission $T=H_{x \text { refr }} / H_{x \text { inc }}$ coefficients, we can write

$$
\begin{aligned}
1-R & =T, \\
-\rho_{0} R & =\chi_{y} T, \\
-\mu_{0} \rho_{0} & =\varepsilon_{z y} \chi_{y} T+\varepsilon_{z z} \chi_{z} T+\frac{\sigma}{H_{x \text { inc }}},
\end{aligned}
$$

instead of (23). Now it can be found from (24) that

$$
\begin{aligned}
R= & \frac{k_{z} \varepsilon_{z z}+k_{y} \varepsilon_{y z}}{\rho_{0} \omega\left(\varepsilon_{y y} \varepsilon_{z z}-\varepsilon_{y z} \varepsilon_{z y}\right)-k_{z} \varepsilon_{z z}-k_{y} \varepsilon_{y z}}, \\
T= & -\frac{\rho_{0} \omega\left(\varepsilon_{y y} \varepsilon_{z z}-\varepsilon_{y z} \varepsilon_{z y}\right)}{\rho_{0} \omega\left(\varepsilon_{y y} \varepsilon_{z z}-\varepsilon_{y z} \varepsilon_{z y}\right)-k_{z} \varepsilon_{z z}-k_{y} \varepsilon_{y z}}, \\
\sigma= & \frac{\rho_{0}\left[\left(\varepsilon_{y y} \varepsilon_{z z}-\varepsilon_{y z} \varepsilon_{z y}\right)\left(\omega \rho_{0} \varepsilon_{0}-k_{y}\right)-\varepsilon_{0}\left(k_{y} \varepsilon_{y z}-k_{z} \varepsilon_{z z}\right)\right]}{\rho_{0} \omega\left(\varepsilon_{y y} \varepsilon_{z z}-\varepsilon_{y z} \varepsilon_{z y}\right)-k_{y} \varepsilon_{y z}-k_{z} \varepsilon_{z z}} \\
& \cdot H_{x \text { inc. }}
\end{aligned}
$$

The analysis of the obtained expression shows that the parts of an electromagnetic wave transmitting in a medium and reflected from the interface depend on the parameters of the medium and do not depend on frequency. Indeed, $k_{y}$ and $k_{z}$ include $\omega$ in the first degree. Therefore $\omega$ can be reduced in (25). The frequency dependence is observed only in the case of frequency dispersion of the dielectric permittivity [5]. Moreover, the coefficients in (23) depend on the inclination angle of the anisotropy axis. At the interface, there is electrical charge which is a result of the polarization of the molecules in the electromagnetic field.

\section{Numerical Results}

In this section, the numerical example of the extraordinary electromagnetic wave behavior in the considered particular case is presented. We consider the quartz medium with the typical parameters $\varepsilon_{11}=4.5, \varepsilon_{33}=4.6[7,8]$ and without frequency dispersion. Only the extraordinary wave is studied because the behavior of an ordinary wave is analogous to the case of an isotropic medium. Indeed the wavenumbers of the forward and backward waves are the same as the ordinary wave.

In Figure 4(a), the dependence of the transmission angle of the wave front on the inclination angle of the anisotropy axis is presented. In Figure 4(b), the dependence of the power transmission angle on the inclination angle of the anisotropy axis is presented. It is seen that the angles have low dependence on $\beta$ (the reflection angle is $\alpha_{\text {refr }}=27.799^{\circ} \div 28.12^{\circ}$ 


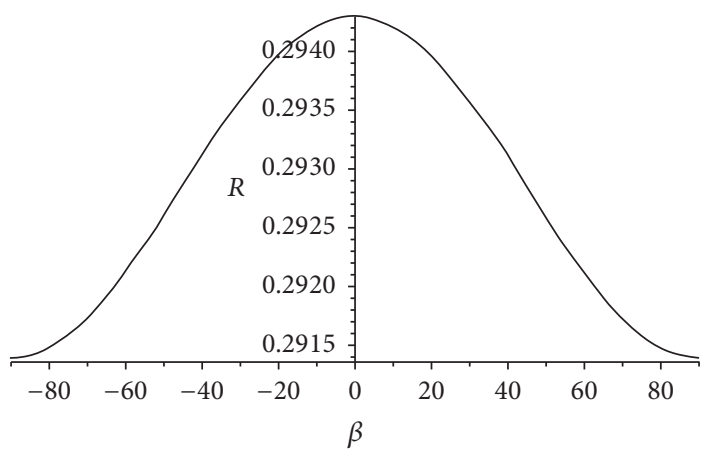

(a)

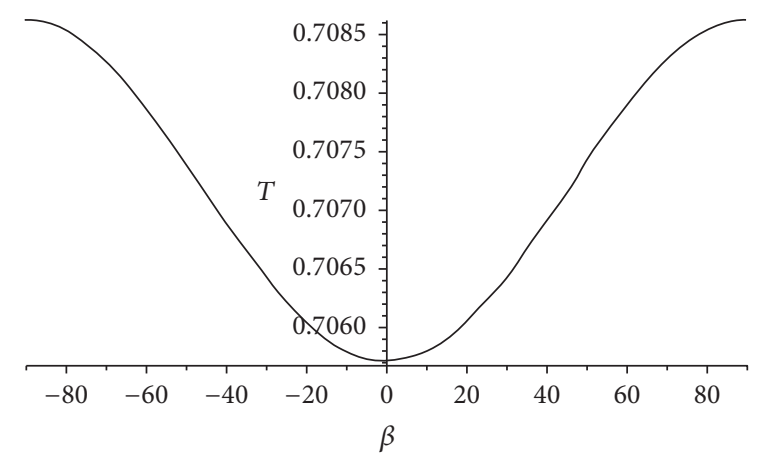

(b)

FIgURE 5: The dependencies of the transmission and reflection coefficients on the inclination angle.

if the inclination angle is $\beta=-180^{\circ} \div 180^{\circ}$ ), and the directions of the wave front propagation and power propagation are different.

It has been also verified by numerical calculations that there is no frequency dependence of these angles. Dependence can be seen only if frequency dispersion of the constitutive parameters $\varepsilon_{11}, \varepsilon_{33}$ is taken into account. For example, it can be plasma medium in a resonance domain.

The dependencies of the transmission and reflection coefficients for the medium parameters given above are presented in Figure 5. It is seen from Figure 5 that the maximum transmission corresponds to the tangential orientation of the anisotropy axis and the maximum reflection corresponds to the normal orientation of the anisotropy axis. Moreover, the reflection and transmission coefficients are symmetrical functions with response to the normal orientation of the anisotropy axis in spite of the fact the normal wave vector constant is asymmetric.

We must also note that $T$ is not the transmission coefficient in the classical meaning [4]. Indeed a transmitted wave power also depends on the relation between the components $E_{x}, H_{y}, H_{z}$.

\section{Conclusions}

In this paper, we consider the behavior of the harmonic plane wave propagating parallel to the interface between vacuum and a uniaxial anisotropic medium. It is shown that the wave propagating in an anisotropic medium has both the normal component of the wave vector and the normal component of the Poynting vector.

It is also proved that in this case the reflected wave should propagate perpendicularly to an interface. At the same time, the reflected wave is absent for an ordinary wave. Moreover, in this case, the reflection and transmission coefficients do not depend on the frequency if frequency dispersion of material parameters is absent.

It is important to dwell on the practical applications of this phenomenon. In particular, we note that it must be taken into account the calculation and design of microwave and optical communication systems. Furthermore, penetration effect enables the creation of new microwave and optical devices such as a power divider [1], a frequency detector with isolator properties [9], and a duplex isolator [10, 11] for telecommunication systems.

\section{Conflicts of Interest}

The authors declare that there are no conflicts of interest regarding the publication of this manuscript.

\section{References}

[1] K. Vytovtov and L. Mospan, "Penetration effect in gyrotropic slab: theory and applications," Journal of the Optical Society of America A: Optics and Image Science, and Vision, vol. 29, no. 6, pp. 877-882, 2012.

[2] K. Vytovtov and S. S. Zouhdi, "Exotic reflection of plane waves by anisotropic structures," in Proceedings of the Metamaterials'2012: The 6th International Congress on Advanced Electromagnetic Materials in Microwaves and Optics, Metamaterials, St. Petersburg, Russia, 2012.

[3] S. A. Andilevko, "A hydrodynamics model of super-deep penetration effect," Journal de Physique IV, vol. 7, pp. 729-734, 1997.

[4] M. Born and E. Wolf, Principles of Optics, 7th Edition, Cambridge, UK, 1999.

[5] T. H. Stix, Waves in Plasma, Springer Science \& Business Media, Media, 1992.

[6] K. Shoemake, "Animating rotation with quaternion curves," in Proceedings of the 12th Annual Conference on Computer Graphics and Interactive Techniques SIGGRAPH '85, pp. 245-254.

[7] L. Ferrari, C. Wu, D. Lepage, X. Zhang, and Z. Liu, "Hyperbolic metamaterials and their applications," Progress in Quantum Electronics, vol. 40, pp. 1-40, 2015.

[8] D. A. Robinson, S. B. Jones, J. M. Wraith, D. Or, and S. P. Friedman, "A review of advances in dielectric and electrical conductivity measurement in soils using time domain reflectometry," Vadose Zone Journal, vol. 2, no. 4, pp. 444-475, 2003.

[9] K. Vytovtov, V. Gnatushenko, and S. Zouhdi, “Terahertz range double direction isolator based on stratified antiferromagneticdielectric structures. Theoretical investigation," Elektronika, vol. 2, pp. 75-78, 2015.

[10] K. Vytovtov, S. Zouhdi, R. Dubrovka, and V. Hnatushenko, "The terahertz controlled duplex isolator: physical grounds 
and numerical experiment," International Journal of Microwave Science and Technology, vol. 2016, Article ID 1468508, 7 pages, 2016.

[11] I. O. Barabanov, N. S. Maltseva, and E. A. Barabanova, "Switching cell for information transmission optical systems," in Proceedings of the 2016 International Conference on Actual Problems of Electron Devices Engineering APEDE 2016, pp. 343347, September 2016. 


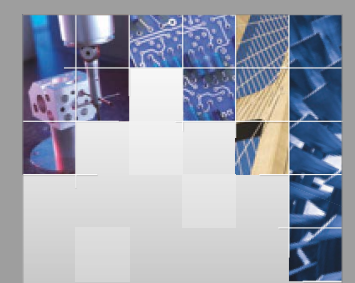

\section{Enfincering}
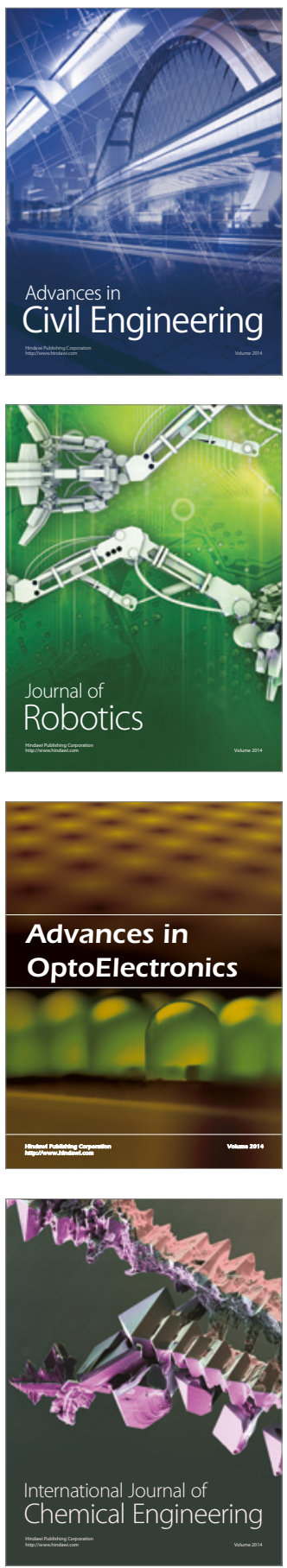

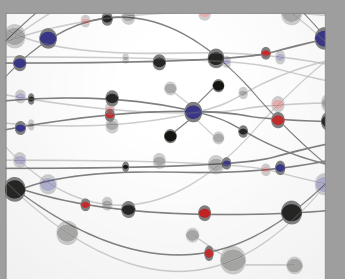

The Scientific World Journal

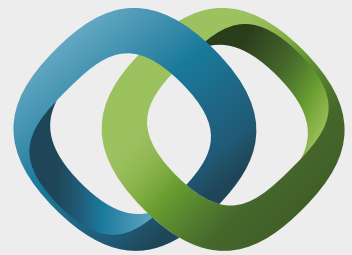

\section{Hindawi}

Submit your manuscripts at

https://www.hindawi.com
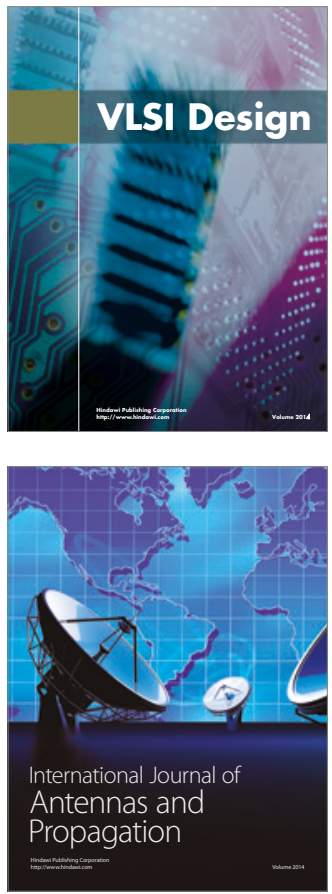

\section{Rotating}

Machinery
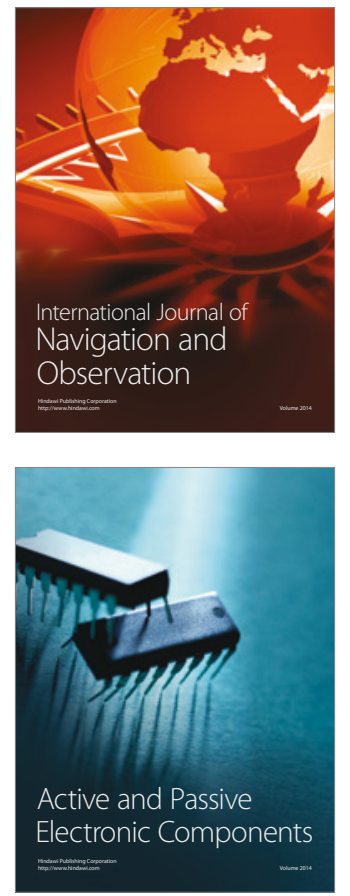
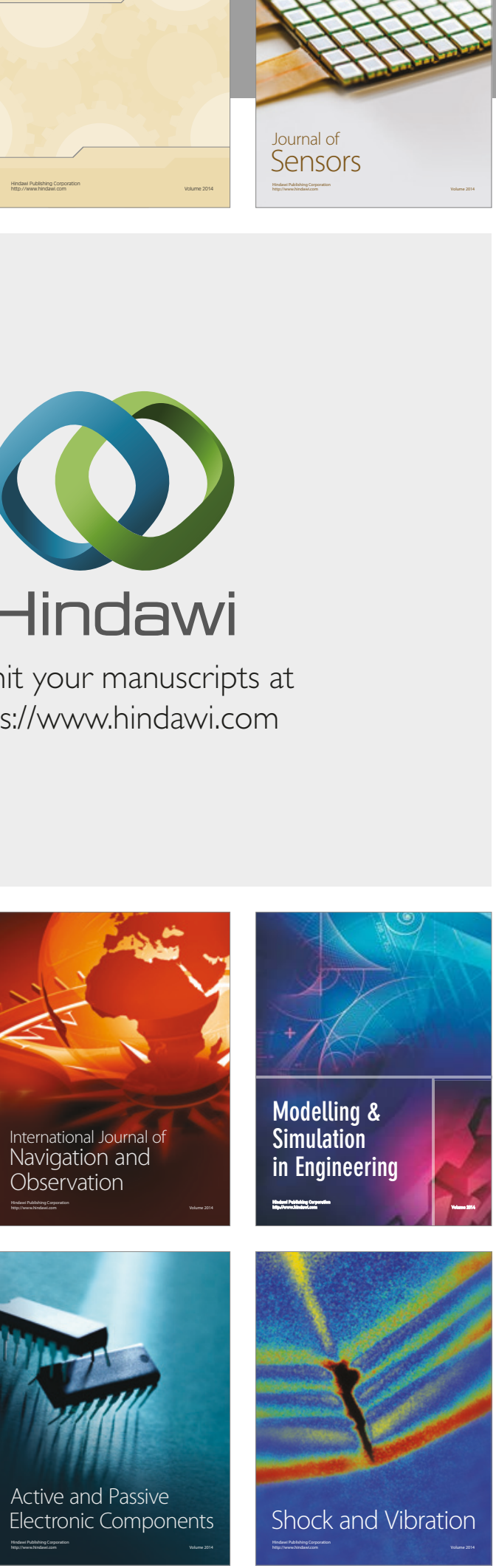
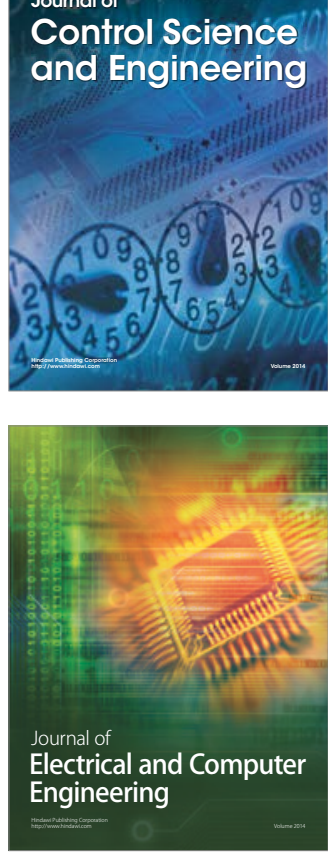

Distributed

Journal of

Control Science

and Engineering
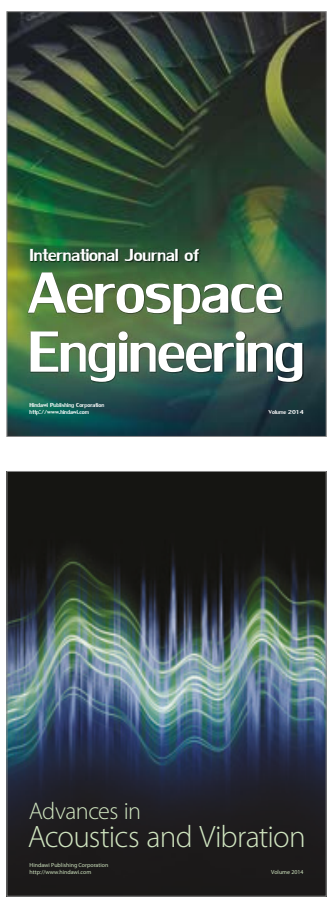

Sensor Networks 